Research Letter

\title{
Immunohistochemical characteristics in diagnostics of prostate diseases
}

\author{
Vladimir M. Popkov, Galina N. Maslyakova, Elena S. Voronina \\ Saratov State Medical University n.a. V.I. Rasumovsky, Saratov, Russia
}

Received 12 January 2013, Accepted 30 January 2013.

(C) 2013, Popkov V.M., Maslyakova G.N., Voronina E.S.

(C) 2013, Russian Open Medical Journal

Abstract: The comparative assessment of molecular markers expression during prostate gland diseases of dishormonal and tumorous nature was carried out and peculiarities of histochemical characteristics have been revealed on the basis of physical examination of 57 patients, which have been treated at the Research Institute of Fundamental and Clinical Uronephrology of Saratov State Medical University n.a. V.I. Rasumovsky.

Keywords: prostate diseases, immunohistochemical markers

Cite as Popkov VM, Maslyakova GN, Voronina ES. Immunohistochemical characteristics in diagnostics of prostate diseases. Russian Open Medical Journal 2013; 2: 0109.

Correspondence to Prof. Galina N. Maslyakova. Address: Department of pathological anatomy, Saratov State Medical University n.a. V.I. Rasumovsky, 112, Bolshaya Kazachiya str., Saratov, 410012, Russia. Tel.: +7 (8452) 669751. E-mail: gmaslyakova@ yandex.ru

\section{Introduction}

According to official statistics, in recent years the number of registered patients with diseases of the prostate in Russia was progressively increased by $56.6 \%$, the growth of morbidity was from 717,800 patients in 2002 to 1,124,417 people in 2008 [1]. The index of reported cases per 100 thousand of adult male population has also increased by $52.9 \%$ - from 1408.5 to 2154.1 during these years. Maximum values of the incidence of prostate disease per 100 thousand adult male populations by regions were recorded in Moscow and St.-Petersburg [1]. The share of prostate tumors in the structure of oncourological diseases in 2008 was $9.7 \%$ [2].

Prostate cancer is the second place in leading cause of death from cancer in men [4]. Prostate cancer is more common in older men. Consequently, it is a big problem in developed countries, where the percentage of elderly men is higher. For example, in developed countries, prostate cancer is about $15 \%$ of cancers in men, whereas in developing countries $-4 \%$ [5].

Triad of pathological processes, developing in the prostate, such as: chronic prostatitis, benign prostatic hyperplasia and prostate cancer - are dominating group of diseases in older men compared to other any clinical entity. These diseases share common pathogenic mechanisms and a similar clinical picture, which greatly complicates the process of early diagnosis of prostate cancer. In this regard, there is the need to find, develop and implement new criteria of early diagnosis of different variants of the tumor in the prostate gland. This can be done with the use of molecular diagnostic markers and, above all, the method of immunohistochemistry.

In clinical recommendations for urology, issued by the European Association of Urology (2010), the criteria for diagnosis at the molecular level are not available. Although it is well known that disturbance of molecular mechanisms plays an important role in the pathogenesis of tumors. The cause of these disorders may be the inflammatory and dishormonal prostate

From our point of view, the possibility of early detection of prostate cancer symptoms is to establish prognosis of development of inflammatory or dishormonal prostate diseases by carcinogenic path.

The aim of this work was a comparative evaluation of expression of molecular markers in prostate diseases of inflammatory and neoplastic dyshormonal nature.

\section{Material and Methods}

The examination of 57 patients was treated at the Institute of Fundamental and Clinical Uronephrology of Saratov State Medical University n.a. V.I. Razumovsky. Some groups of patients were allocated: the first group - 22 patients with benign prostatic hyperplasia (BPH) and biopsy revealed prostatic intraepithelial neoplasia (PIN) high, the second group - 20 patients with prostate cancer, the third group - 15 patients with severe morphological signs of chronic inflammation.

For the diagnosis of prostate diseases, including prostate cancer screening, a combination of methods such as analysis of the patient's complaints, the study overall level of prostate specific antigen serum; digital rectal examination of the prostate, transrectal ultrasound of the prostate and seminal vesicles with the definition of residual urine; pathological verification prostate with polifocal prostate biopsy under transrectal ultrasound guidance (if necessary) and surgical specimens was used. The indications for prostate biopsy were index of prostate-specific antigen in serum above $4.0 \mathrm{ng} / \mathrm{ml}$, a suspicion of prostate cancer by digital rectal examination or ultrasound scan. Prostate biopsy was carried out from 8-12 points (average 10 points) and the 
sighting from the sections of the parenchyma where the prostate cancer was suspected.

Histochemical and immunohistochemical reactions were performed on serial paraffin sections using a streptavidin-biotin method.

16 commercial monoclonal antibodies were used for immunohistochemical study. They were separated into groups of functional significance: markers of proliferative activity (Ki-67, PCNA); regulators of the cell cycle (p53 and p63), markers of apoptosis (bcl-2, BAX), EGF receptor growth (EGFR); cytokeratin 34 $\beta E 12$, markers of hormonal activity (AR, ER); marker of cell-cell interactions (E-cadherin), prostate specific antigens (PSA, PSMA, AMACR); marker of neuroendocrine cells (Chr); markers of membrane receptors (lectins wheat and almond). The results of immunohistochemical studies (Ki 67, PCNA, p53, p63, the expression of androgen receptor and estrogen-nuclear expression) were evaluated by using Histochemical score $\mathbf{H s}=\mathbf{\Sigma} \mathbf{P} \mathbf{x} \mathbf{I}$ (I-staining intensity, $\mathrm{P}$ - the percentage of stained cells). Only a qualitative assessment was used in the reaction with Amacr, $B c 12$, and $E$ cadherin, VEGF, PSMA, PSA, BAX, Chr (cytoplasmic and membrane expression) used (+ weak expression, ++ moderate expression, +++ strong expression).

Treatment of the material was carried out on an automated system of morphological images "Ariol» (Genetix, USA). Quantitative evaluation of the results of immunohistochemical reactions was performed in micrographs obtained with the locking system of microscopic images. Data analysis was conducted using program of statistical analysis "Statistisa 6.0".

\section{Results and Discussion}

After making statistical treatment of the material by the methods of parametric and non-parametric statistics, we found a significant correlation between expression of molecular markers, character pathology and patient age. It suggests the possibility of using of these indicators as prognostic and diagnostic factors. It was found that expression of proliferative marker Ki-67 and PCNA was observed in all studied groups. The number of cells, expressing Ki-67 was the lowest in comparison group of patients $(\mathrm{Hs}=10)$ and increased in patients with $\mathrm{BPH}(\mathrm{Hs}=25)$. The highest expression of Ki-67 was obtained in patients with prostate cancer $(H s=110)$. The intensity of expression increases with the increasing of number of expressing cells.

The same trend was observed in the determination of the degree of expression of the marker PCNA. In the comparison group proliferative activity of cells was three times lower $(\mathrm{Hs}=30)$ than in patients with prostate cancer $(\mathrm{Hs}=98)$. It was a statistically significant correlation between the degree of PCNA expression and the age of patients $(p<0.05)$ in patients with inflammatory diseases and benign dyshormonal prostatic dysplasia. With increasing of patient age the degree of expression of this marker was reduced. In patients with prostate cancer statistically significant correlation between the intensity of expression and age was not observed.

In defining of marker of cell cycle regulators P53 and P63 positive expression of p53 was observed in $90 \%$ of patients with prostate cancer. In patients with BPH positive expression was detected in $78 \%$ of cases, in the group of patients with chronic prostatitis it was single. Perhaps this is due to the fact that at chronic inflammation macrophage and phagocytic cells produce large amounts of free radicals, which are directly or indirectly violate the structure and functional activity of proteins, in particular, p53, and also contribute to the mutagenic activity of the cells. In the recent literature the information appeared that the marker P63 is expressed in stem cells of the basal epithelium and this indicates the presence of cells with great potential for self-renewal and differentiation. In our research p63 showed positive expression in the basal cells of glands in patients of all three groups. However, in patients with chronic prostatitis such cells were preserved, but their number was drastically reduced in the group of patients with cancer.

From three prostatic specific markers AMACR (alpha metilatsilCoA racemase) showed most demonstrative picture, in agreement with other authors [3]. Detection of AMACR in body fluids has significant limitations in connection with its low specificity. This marker is expressed by some malignant tumors of other localizations (eg, breast or pancreas). At immunohistochemical study AMACR showed a high specificity when expressed in a group of patients with prostate cancer (+++ strong expression). However, it was found a decrease of the intensity of expression with age. Undoubtedly, this fact must be taken into account in the histological study.

Prostate specific membrane antigen is an integral membrane protein with enzymatic activity. In our material, it was noted the active membrane expression of PSMA in prostate cancers (+++ strong expression). In patients with BPH weak cytoplasmic expression was predominated and the phenomenon of moving expression of the marker from the cytoplasm to the cell membrane was marked. This may be a sign of an early stage of carcinogenesis. The same marker showed pronounced correlation dependence between degree of the expression and the patient's age: the intensity of expression decreased with increasing age.

PSA level in normal prostate tissue is a million times greater than the rates in the serum. In our material PSA is defined by immunohistochemical method in all the structures of both normal and hyperplastic and neoplastic prostate. The intensity of the histochemical reaction was not correlated with the concentration of in the peripheral blood. In addition, the expression of PSA in patients with prostate cancer decreased regardless of patient age.

It was known that PSA is an androgen-dependent enzyme. In our material, we have also received the correlations between the decline in PSA levels and the amount of androgens. As for estrogen, we have not established correlation between their expression and the character of disease.

It was found that androgen receptors are absent in the neuroendocrine cells, thus reducing of the number of androgen, including androgen blockade, may lead to preferential growth of these cells. Neuroendocrine cells have low proliferation and exercise their effects through the secretion of neuropeptides. There are suggestions that neuroendocrine cells contribute to the development of androgen-independent prostate cancer through paracrine effects of growth factors. Our research was showed that the number of neuroendocrine cells in the study groups was not statistically different from each other. However, it was the interrelation between the marked expressions of chromogranin A in patients with prostate cancer after maximal androgen blockade. We suggest that increasing the number of neuroendocrine cells in the biopsy may be a sign of androgen-refractory prostate cancer.

It is known that in normal prostate tissue the apoptotic cell death rate is of $1-2 \%$ per day. This process is balanced by cell proliferation. In the study of markers of apoptosis their natural behavior of tumors was noted in the study groups: increased expression of apoptosis blocker of bcl-2 and decreased expression 
of the activator BAX, which points to a regular shift of carcinogenesis towards in the direction of proliferative processes.

Epithelial cadherin (E-cadherin) is a calcium-dependent adhesion molecule, linking cells by homotypic interactions. Suppression or loss of its function is associated with invasive and aggressive behavior of different types of human tumors. At comparative analysis of the content of this protein in the treatment groups it was found that their expression decreases significantly in patients with prostate cancer. In none of the cases we checked the absence of this protein, which would indicates a poor prognosis of the disease and the possible appearance of fast metastases.

Expression of cytokeratin $34 \beta \mathrm{E} 12$ was intensive in the tissues of the prostate gland with non-neoplastic processes and it reduces in prostate cancer.

In the literature we did not meet the data about using of lectins as a marker of prostate cancer diagnosis, which marker showed high specificity and sensitivity in our study. There is a certain analogy between the methods lectinohistochemistry and immunohistochemistry. But, in contrast to antibodies, lectins interact only with carbohydrate determinants of biopolymers and have a much lower cost of the study.

Set of carbohydrates of cancer cells is very different from that of normal cells and can serve as a tumor marker. In our study we used 5 different lectins, but only two of them - WCA (lectin wheat germ) and LBA (lectin of Laburnum anagyroides) - showed significant results. Their expressions in patients with prostate cancer were significantly higher than in other diseases, and did not depend on the age of the patients.

Table. The intensity of expression of immunohistochemical markers in hyperplastic, neoplastic processes, and inflammation of the prostate

\begin{tabular}{lcccc}
\hline Markers & $\begin{array}{c}\text { Group of } \\
\text { comparison }\end{array}$ & $\begin{array}{c}\text { Diffuse } \\
\text { hyperplasia of } \\
\text { the prostate }\end{array}$ & Cancer & Prostatitis \\
\hline Ki 67 & $\mathrm{Hs}=10$ & $\mathrm{Hs}=25$ & $\mathrm{Hs}=110$ & $\mathrm{Hs}=30-40$ \\
P53 & $\mathrm{Hs}=26$ & $\mathrm{Hs}=80$ & $\mathrm{Hs}=103$ & $\mathrm{Hs}=84$ \\
P63 & $\mathrm{Hs}=91$ & $\mathrm{Hs}=84$ & $\mathrm{Hs}=17$ & $\mathrm{Hs}=83$ \\
Amacr & Unit cells & Unit cells & +++ & Unit cells \\
PSMA & ++ & + & +++ & ++ \\
PSA & +++ & +++ & ++ & +++ \\
Estr & $\mathrm{Hs}=30$ & $\mathrm{Hs}=36$ & $\mathrm{Hs}=38$ & $\mathrm{Hs}=27$ \\
Chrom & + & + & ++ & + \\
Bcl2 & + & ++ & +++ & + \\
BAX & ++ & ++ & + & ++ \\
E cad & +++ & ++ & + & +++ \\
CK34ßE12 & +++ & +++ & + & +++ \\
Lect.WCA & + & + & +++ & + \\
\hline
\end{tabular}

$\mathrm{Hs}$ - histochemical score.

\section{Conclusions}

1. For statistical analysis the high degree of significance correlation dependence between the groups of patients with prostatitis, BPH and prostate cancer was observed in the expression of markers of AMACR, Ki-67, P53, P63, cytokeratin $34 \beta E 12$, the lectin wheat germ and bcl-2. Correlation with moderate significance was observed at expression of lectin of Laburnum anagyroides, E-cadherin and BAX.

2. It was noted a reduction of the intensity of expression of markers of tumor growth in the elderly - PSNA, PSMA, AMACR and chromogranin. Likely, this is due to reduced of metabolic activity and changes in hormonal levels.
Conflict of interest: none declared.

\section{Reference}

1. Apolikhin OI, Sivkov AV, Beshliev DA, Solntseva TV, Komarova VA. Analysis of urological morbidity in the Russian Federation according to official statistics. Experimental and Clinical Urology 2010; (1): 4-10. [Article in Russian]

2. Malignant neoplasms in Russia in 2008 (morbidity and mortality). V.I. Chissova, V.V. Starinskogo, G.V. Petrova Eds. Moskow, Russia, 2010. [Text in Russian]

3. Pozharissky KM, Leenman EE, Arzumanov AA. Achievements in morphological diagnosis of prostatic cancer: alpha-methylacylcoenzyme-A-racemase - a new marker of malignant cell transformation. Archives of Pathology (Arkhiv Patologii) 2005; 67(5): 15-19. (PMID: 16323473) [Article in Russian]

4. Jemal A, Siegel R, Ward E, Hao Y, Xu J, Murray T, Thun MJ. Cancer statistics, 2008. CA Cancer J Clin 2008; 58(2): 71-96. (PMID: 18287387) (doi: 10.3322/CA.2007.0010)

5. Parkin DM, Bray FI, Devesa SS. Cancer burden in the year 2000: the global picture. Eur J Cancer 2001; 37 (Suppl 8): S4-66. (PMID: 11602373)

\section{Authors:}

Vladimir M. Popkov - MD, D.Sc., Rector of Saratov State Medical University n.a. V.I. Rasumovsky, Saratov, Russia;

Galina N. Maslyakova - MD, D.Sc., Professor, Head of the Department of pathological anatomy, Saratov State Medical University n.a. V.I. Rasumovsky, Saratov, Russia;

Elena S. Voronina - MD, PhD, Assistant Professor, Department of pathological anatomy, Saratov State Medical University n.a. V.I. Rasumovsky, Saratov, Russia. 